Voix et Images

volxetimages

\title{
L'espace de nos fictions : quelques réflexions sur nos deux cultures
}

\section{Patricia Smart}

Volume 10, numéro 1, automne 1984

Littérature canadienne-anglaise

URI : https://id.erudit.org/iderudit/200455ar

DOI : https://doi.org/10.7202/200455ar

Aller au sommaire du numéro

Éditeur(s)

Université du Québec à Montréal

ISSN

0318-9201 (imprimé)

1705-933X (numérique)

Découvrir la revue

Citer cet article

Smart, P. (1984). L'espace de nos fictions : quelques réflexions sur nos deux cultures. Voix et Images, 10(1), 23-36. https://doi.org/10.7202/200455ar d'utilisation que vous pouvez consulter en ligne.

https://apropos.erudit.org/fr/usagers/politique-dutilisation/ 


\title{
L'espace de nos fictions: quelques réflexions sur nos deux cultures
}

\author{
par Patricia Smart, Université Carleton
}

Si la situation de tension est inhérente à la dialectique et que celle-ci oppose deux pôles adverses qui se révèlent l'un à l'autre dans une situation progressive, c'est commettre un acte de lèse-dialectique que de nier que le Canada est un cas dialectique bien défini, où se confrontent deux cultures. Il est plus logique d'aller dans le sens de cette opposition critique des deux cultures, si l'on veut en arriver à comprendre quelque chose à la situation canadienne, que de désaxer la dialectique historique dans laquelle le Canada français se trouve impliqué...

Hubert Aquin, «La Fatigue culturelle du Canada français»

mais la fiction est-elle un genre ouvert (Québec) ou une façon de négocier avec la tradition (Canada)? je sais. un compromis. optons pour le style hybride. geste bien féminin! tissons une histoire pardessus les trous.

Gail Scott, «A Story Between Two Chairs », texte présenté au colloque NBJ sur la modernité, le 29 février 2984.

Qu'un numéro spécial de Voix et Images soit consacré à la littérature canadienne de langue anglaise doit sûrement constituer un événement et un point de repère dans l'histoire des relations entre nos deux cultures. ${ }^{*}$ Qui eât cru, même il y a cinq ans, qu'il y aurait assez de confiance et de dynamisme des deux côtés pour permettre ce regard plus ou moins dégagé

* Ce texte est l'adaptation de «Some Reflections on Our Two Cultures», discours inaugural prononcé au premier colloque de l'Association nordique des études canadiennes, à Aarhus, Danemark, le 30 avril 1984. La traduction du texte original est de Dorothy Howard. 
sur le Canada de la part d'une revue littérature québécoise? Cette nouvelle confiance a été gagnée lentement et péniblement par la génération nationaliste (Dennis Lee, Margaret Atwood, Robin Mathews, Margaret Laurence, Al Purdy) tant au Canada qu'au Québec. Dans les miroirs placés devant eux par les écrivains de cette génération, les deux peuples ont découvert que malgré les apparences, malgré l'envahissement culturel étranger, ils avaient chacun un projet collectif. Les miroirs ont tendance à devenir prisons, toutefois, à moins qu'ils ne soient traversés; et c'est ce qui semble en train de se produire dans chacune des deux cultures depuis 1980, dans la multiplication des rencontres et des colloques littéraires internationaux tant au Canada et au Québec qu'à l'étranger.

«Pour fleurir», disait Gaston Miron dans une entrevue récente, «la poésie a besoin d'une terre, d'un espace, d'une lumière, d'un milieu où elle peut s'enraciner, et de beaucoup d'amitié. " 1 Je voudrais amorcer ici une réflexion sur cette terre, ces espaces, ces milieux où nos deux littératures ont pris racine, et faire quelques suggestions quant à la façon différente dont les écrivains des deux cultures ont habité les territoires du réel, de l'imaginaire et du langage.

Déjà des ambiguïtés, des façons différentes d'habiter l'espace et le langage: de qui parlons-nous? Rattachés l'un à l'autre depuis la bataille où Montcalm et Wolfe ont tous deux perdu la vie, nous avons transposé nos batailles et nos frontières psychologiques jusque dans les noms que nous nous sommes donnés. Depuis les appellations clairement démarquées d'Anglais et de Canayens utilisées par les deux peuples jusqu'en 1840, nous avons fait le tour de toute une gamme de noms composés ou simples cherchant à cerner notre relation et notre sentiment d'appartenance à un territoire souvent contesté.

L'Histoire joue pour beaucoup dans les consciences québécoise et canadienne-anglaise : à la différence des Américains, nous ne nous sommes jamais pris pour de nouveaux Adam et Eve ouvrant en territoire vierge et en rupture totale avec la tradition. Ainsi, dans ce pays où le bilinguisme officiel - le rêve de notre premier ministre démissionnaire - a rencontré une résistance assez forte pour empêcher l'Assemblée législative du Manitoba de siéger l'automne dernier, il est tentant de revenir à l'idée consacrée par la tradition de «deux cultures fondatrices», la française et l'anglaise. Ce terme est cependant rejeté par plusieurs Canadiens de l'Ouest, dont les ancêtres sont arrivés bien après le jeu d'échecs qui a scindé le pays en deux; il est également rejeté, de façon plus convaincante encore, par les autochtones.

1. Entrevue à Radio-Québec, dans la série «Profession : écrivain», le ler avril 1984. 
Parler tout simplement de cultures canadienne-anglaise et canadiennefrançaise a l'avantage pratique d'inclure les membres importants de chaque culture qui vivent et écrivent dans le territoire de l'Autre, tels Gabrielle Roy, Antonine Maillet, Mordecai Richler ou Leonard Cohen pour n'en nommer que quelques-uns, mais les événements ont donné une saveur décidément fédéraliste à ces termes. Quitte à reléguer ces écrivains à un territoire sans nom, nous avons adopté les termes "canadien» et «québécois» pour désigner les deux cultures. Même du côté canadien-anglais, cette pratique devient de plus en plus courante, du moins chez les universitaires. Notons tout de même l'ambiguitté de ces termes tels qu'utilisés au Canada anglais, où il n'est jamais clair si l'on se réfère à la situation de la partie (Québec) dans le tout (Canada) ou à deux territoires de l'imagination qui s'excluent mutuellement. À ce stade de nos histoires, il semble plus réaliste de parler des traditions culturelles "canadienne-anglaise " et "québécoise".

Qu'en est-il de ces deux traditions culturelles? Comment se sont-elles regardées? Y aurait-il entre elles des bases de comparaison valables? Etant donné la proximité historique dans laquelle nous avons vécu, le nombre relativement restreint d'études comparatives a sans doute une explication idéologique. Pendant longtemps, la frontière entre les deux cultures a été inexistante pour les critiques canadiens-anglais : il y a des actes qui se réalisent plus facilement sur papier que dans la réalité, comme celui d'assimiler la tradition québécoise au grand tout canadien. Quant au petit nombre d'études comparatives provenant du Québec, il semble s'agir tout simplement d'autres priorités : le Québec lui-même, mais aussi l'Europe, les ÉtatsUnis, l'Amérique latine. N'importe où, pourvu que ce ne soit pas le Canada anglais, serait tenté de penser le pauvre observateur anglophone! Si par hasard il tombe sur un article traitant du Canada anglais, il peut s'attendre à le voir précédé d'un titre comme celui du récent numéro de la revue Nuit blanche: «Le Canada existe-t-il ? 2». Il semble que culturellement le Canada ait été perçu par le Québec comme une région des États-Unis 3 - belle revanche! C'est presque comme si dans le domaine culturel le rapport entre dominateur et dominé était renversé : le Canadien anglais enviant et admirant la passion, la sophistication théorique, le caractère militant et collectif qu'il perçoit dans la littérature québécoise, tout en restant hésitant à propos de son propre discours et culpabilisé par les rapports qui existent dans le domaine politique.

2. Nuit blanche, No. 11, déc. 1983 / janv. 1984.

3. Voir par exemple Robert-Guy Scully, «La culture du Canada anglais», le Devoir, 10 mars 1973. 
Les critiques qui se sont penchés sur les deux littératures ont proposé un certain nombre de symboles géométriques pour illustrer leur relation des lignes parallèles, l' «horizontalité» du Canada anglais et la "verticalité" " du Québec, un escalier en spirale, une ellipse, une double hélice, un treillis 4 . Mais comme la critique elle-même, surtout la critique comparative, ces figures demeurent abstraites et tendent à négliger les impératifs historiques qui ont défini nos deux cultures et les rapports qui se sont tissés entre elles. Une impression plus claire des images que nous projetons se dégage à mesure que nous parcourons nos littératures. Nous nous retrouvons très vite dans le domaine des stéréotypes, mais on se rappellera que les stéréotypes prennent racine dans une réalité imposée par l'histoire et que nos écrivains s'amusent, justement dans ce domaine, à nous révéler à nous-mêmes, proposant ainsi de nouvelles perceptions.

Dans les littératures québécoise et canadienne-anglaise d'avant les années soixante, il y a vraiment lieu de parler de stéréotypes, ceux-ci n'éclatant presque jamais que pour explorer le caractère ambivalent de la relation entre les deux cultures. Dans le roman québécois, de la Terre paternelle à Maria Chapdelaine, Menaud, maitre-draveur, Trente arpents, Bonheur d'occasion, l'Anglais fait figure de conquérant, d'envahisseur, d'industriel riche et insensible à la langue et à la culture qu'il détruit par son expansionnisme économique 5 . La littérature canadienne-anglaise, dans la mesure où elle fait entrer la figure du Québécois, présente l'autre face de la même médaille: les bûcherons dans The Man From Glengarry de Ralph Connor, les habitants dans la poésie de William Henry Drummond et même les Canadiens français dans les Two Solitudes de Hugh Maclennan, sont de charmants restes folkloriques d'une culture destinée à disparaître dans la poussée anglo-saxonne vers le progrès.

Dans nos deux littératures depuis 1960, cependant, ces stéréotypes ont commencé à évoluer et à laisser transparaître, parfois de façon assez vertigineuse, le fond caché de soi tel que reflété dans le miroir de l'Autre. Chez Hubert Aquin, Jacques Godbout, Margaret Atwood et Nicole Brossard, il est possible de dégager certaines images qui peuvent éclairer les changements qui se sont effectués dans la relation des deux cultures depuis une vingtaine d'années.

4. Voir Philipp Stratford, "Canada's Two Literatures: A Search for Emblems», Canadian Review of Comparative Literature / Revue canadienne de littérature comparée 6, printemps 1979, p. 131-138; et E.D. Blodgett, Configuration: Essays on the Canadian Literatures, Toronto, ECW Press, 1982, p. 13-38.

5. Récemment une série de chroniques très polémiques par William Johnson dans le Globe and Mail ( 16 nov. $1983-20$ janv. 1984) a analysé cette image de l'Anglais dans la littérature québécoise en soutenant que le stéréotype est «raciste», une invention des intellectuels et du clergé utilisée pour propager un nationalisme qui avait peu d'appui dans le peuple. 
Le regard de l'Autre paralyse, du moins tant que nos propres assises culturelles sont perçues comme fragiles et incertaines. Si l'on en juge par les deux littératures, le Québec s'en est aperçu plus vite que le Canada anglais (du moins, et c'est une précision importante, dans la mesure où les écrivains canadiens-anglais ont même été conscients de l'Autre culture). Le parallélisme est frappant, et le décalage historique significatif, entre deux ouvrages où il est question de ce regard de l'Autre - Prochain épisode d'Hubert Aquin (1965) et Two-Headed Poems de Margaret Atwood (1978). Dans Prochain épisode la fuite et la poursuite de l'Autre constituent la seule intrigue possible, la condition préalable à l'union avec la femmepays aimée et avec soi-même; et elles mènent à la «stase volcanique » d'un désir de fusion mystique identifiée à la mort. Cet Autre est perçu par le narrateur (et c'est un thème auquel je reviendrai) comme puissant et attirant dans sa façon d'habiter l'espace:

... le plaisir d'habiter une maison peut donc ressembler à la complaisance ébahie que j'éprouve dans ce salon ample et majestueux. $H$. de Heutz vit dans un univers second qui ne m'a jamais été accessible, tandis que je poursuis mon exil chaotique dans des hôtels que je n'habite jamais 6 .

L'image que nous donne Aquin de la relation entre les deux cultures en est une de fascination, de tension et enfin de stase, comme dans la sculpture baroque des deux guerriers qui «tendus l'un vers l'autre en des postures complémentaires, sont immobilisés par une sorte d'étreinte cruelle, duel à mort qui sert de revêtement lumineux au meuble sombre " 7 . C'est une image qui reviendra peut-être nous hanter mais qui, en cette atmosphère postréférendaire des années 1980 , semble plutôt révolue.

Une quinzaine d'années après la parution de Prochain épisode, une image plus grotesque et plus carnavalesque nous est proposée simultanément par deux écrivains majeurs des deux cultures. Margaret Atwood, dans son recueil de 1978 Two-Headed Poems, et Jacques Godbout, dans son roman de 1981 les Têtes à Papineau, décrivent le Canada anglais et le Québec comme des jumeaux siamois, rattachés par le cou ou par la tête et sur qui toute chirurgie est impossible. L'image des siamois est peut-être plus désespérée que celle d'Aquin par son sens d'un fatalité biologique, géographique et historique irréductible, d'une farce monstrueuse qui caractérise notre naissance; cependant, elle est aussi plus libératrice dans la mesure ou elle incite à rire et accepter nos natures grotesques mais vraies. L'image de Godbout est, en fait, propre au Québec: ses jumeaux siamois ne sont pas précisément le Québec et le Canada, mais plutôt les côtés "canadien» et "français » de la psyché québécoise, et le conflit entre ces deux «têtes», entre l'anonyme technologie anglo-saxonne et les monstres de famille provenant du passé culturel québécois, est perçu comme un problème interne à la famille québécoise.

6. Prochain épisode, Montréal, CLF, 1965, p. 128.

7. Ibid., p. 127. 
Les Two-Headed Poems d'Atwood présentent la même image de jumeaux siamois, cette fois à deux corps et joints par la tête, représentant un Canada que le poète craint de voir scindé en deux :

Why fear the knife that could sever us, unless it would not cut skin but brain 8 ?

Ce qui est intéressant dans cette suite poétique, c'est le renversement du rapport entre colonisateur et colonisé, et le silence du Canadien anglais qui se trouve emprisonné dans le stéréotype dicté par l'Autre culture. Les Québécois y apparaissent comme une "grande famille heureuse», assise bien sûr autour d'une table de pin, tandis que les Canadiens anglais se savent les voisins indésirés, trop bruyants, noircissant les roses des cours arrière des maisons par la fumée de leurs barbecues. Comment le dialogue est-il possible, ou même l'articulation de sa propre voix, quand on sait que cette voix est entendue par l'Autre comme

a language for ordering the slaughter and gutting of hogs, for counting stacks of cans. Groceries are all you are good for. Leave the soul to us 9.

Malgré l'ironie contrôlée en surface, le poème d'Atwood est un plaidoyer émouvant pour le dialogue, un aveu du sentiment de paralysie éprouvé par le Canadien anglais en face de ce qu'il perçoit comme l'indifférence québécoise :

These words slow us, stumble in us, numb us, who can say even Open the door, without these diffident smiles, apologies?

If I were a foreigner, as you say instead of your second head, you would be more polite...

But we are not foreigners

to each other; we are the pressure on the inside of the skull, the struggle among the rocks for more room, the shove and giveaway, the grudging love the old hatreds 10 .

8. Two-Headed Poems, Toronto, Oxford University Press, 1978, p. 70.

9. Ibid., p. 74.

10. Ibid., p. 70. 
Enfermées dans l'abstraction du regard, du cerveau, de la tête, ces images traduisent toutes une impuissance et un échec similaires à ceux qui ont prévalu dans les tentatives avortées de débat politique entre les deux cultures: "not a debate", selon les mots d'Atwood, «but a duet / with two deaf singers » 11. Mais si le regard devenait toucher? Si la logique patriarcale des frontières faisait place à une voix plus corporelle? L'action subversive exercée par le féminisme dans chacune des deux cultures depuis une décennie doit être prise en considération dans tout effort pour situer ces cultures ou analyser leurs rapports. Elle a déjà produit une série d'échanges entre écrivaines et critiques féministes du Québec et du Canada anglais 12 qui sont, si je ne me trompe, sans précédent dans l'histoire de nos deux cultures. C'est pourquoi, avant de passer à une tentative de différenciation de nos deux traditions culturelles, je propose une dernière image, tirée d'un texte récent de Nicole Brossard, comme emblème possible de nos rapports culturels. Cete représentation de deux femmes se tenant dos à dos mais s'appuyant l'une sur l'autre dépasse l'ancienne fascination du regard pour évoquer l'énergie partagée par deux partenaires autonomes, chacune regardant vers le monde extérieur:

C'est la panique, l'embrouillement, c'est l'impossible romancé du face à face, la jonglerie des corps acrobates, le mur de berlin pour appuyer mes reins, le frisson dans le dos

$$
\text { c'est l'émerveillement : voir partout à }
$$

la fois. Elle peut donc lire dans mes pensées pendant que je lui tourne le dos elle s'étonne de mon étonnement $13 \ldots$

Contrairement aux images géométriques proposées par les critiques et aux images de dialogue avorté proposées par les écrivains précédents, cette image est réelle, incarnée et aimante. Et pourquoi pas une image de deux femmes comme emblème de nos deux cultures, étant donné l'importance des femmes, pour des raisons différentes, tant dans la littérature québécoise que canadienne-anglaise? Pourquoi pas une image corporelle, étant donné l'effritement de la mentalité puritaine / janséniste qui a caractérisé nos deux littératures ces dernières années? Dans l'image de Brossard il y a contact mais non fusion; les identités distinctes sont respectées et l'échange devient

11. Ibid., p. 75.

12. Trois colloques: "la Conférence des femmes-écrivains des Amériques » à l'Université d'Ottawa en mai 1978, «Dialogues», colloque sur la critique féministe à l'Université York en octobre 1981, et «Women and Words/ Les Femmes et les mots " a Vancouver en juillet 1983; et une nouvelle revue, Tessera, où des féministes anglophones - inspirées par leurs compatriotes québécoises - explorent les rapports entre femmes, langage et imaginaire.

13. "Je ais tourner mon corps et faire semblant de la comparer », NBJ 136-137, numéro spécial Révélatrices: femmes et photos, p. 154. 
possible, non pas quand les partenaires se regardent face à face, mais lorsqu'elles se soutiennent mutuellement dans leur position face au monde extérieur. Transposée, elle devient l'image de deux nations, de deux projets, d'un espace contigu mais non commun, d'une frontière où les deux cultures trouvent force et vigueur dans la différence. Cette image traduit bien la relation actuelle entre les cultures canadienne-anglaise et québécoise: impliquées toutes deux dans le processus d'intégration des voix des femmes et du monde extérieur, elles découvrent de nouveaux modes de dialogue dont l'effet subvertit la vieille logique des frontières politiques. N'étant plus rattachées par la tête, elles peuvent enfin explorer d'autres façons de se parler avec une intelligence plus corporelle qui n'exclut ni le coeur ni l'histoire commune de leur devenir.

$\mathrm{Si}$, comme je l'ai déjà suggéré, une relation plus détendue et positive se développe entre nos deux cultures, son effet se fera sûrement sentir dans le domaine de la critique littéraire comparative. Jusqu'ici, à une exception près, les études comparatives parues sous forme de livres ont suivi une approche thématique ou sociologique; leur base idéologique est incontestablement fédéraliste 14. Implacables dans leur recherche dè similarités au niveau des thèmes, des genres littéraires et des systèmes de valeurs des deux cultures, elles ont déformé les deux traditions en négligeant les différences fondamentales de contextes idéologiques et d'influences littéraires. L'exception serait Configuration: Essays on the Canadian Literatures de E. D. Blodgett, paru en 1982, dans lequel l'auteur fait cette déclaration importante:

not only that all literary theory is ideological, but that any literary theory that tries to resolve the problems of nation-states that are at least bilingual in an official sense must be clear about its ideology 15 .

Et plus loin, il complète ainsi :

Any literary framework that assumes equality of status between the two major cultural groups in Canada mistakes the nature of the relationship 16 .

Cependant les essais de Blodgett mettent l'accent sur la pluralité des littératures immigrantes, sur les influences européennes et l'utilisation des genres littéraires plutôt que sur une tentative d'explorer les liens entre les traditions littéraires québécoise et canadienne-anglaise.

14. Par exemple, C. Moisan, L'Âge de la littérature canadienne, Montréal, HMH, Coll. "Constantes", 1969; R. Sutherland, Second Image: Comparative Studies in Canadian Literature, Toronto, New Press, 1971 et The New Hero: Essays in Comparative Quebec / Canadian Literature, Toronto, Macmillan, 1977.

15. Blodgett, op. cit., p. 32.

16. Loc. cit. 
Comme mode de comparaison qui pourrait permettre de différencier nos deux cultures, je proposerais une approche centrée sur les façons dont les écrivaines et les écrivains du Québec et du Canada anglais ont habité l'espace: les territoires du réel, de l'imaginaire et du langage. Un commentaire d'un ami québécois qui avait tenté de lire l'ouvre de Margaret Laurence a contribué à cristalliser l'idée que j'avais de notre différence fondamentale. "J'ai essayé d'entrer dans ses romans », m'a-t-il dit, «mais ils sont trop réels. » Implicite dans cette remarque, je crois, était le questionnement suivant: Où est la fantaisie? le rêve? l'excursion aux frontières de la folie à laquelle ma propre culture m'a habitué? Où est l'explosion langagière qui subvertit la relation référentielle de la littérature au réel et qui a donc des conséquences subversives dans la réalité elle-même?

Il n'est pas dans mon intention de nier l'importance des écrivain(e)s «post-modernes» du Canada anglais, tels Robert Kroetsch, Rudy Wiebe, Daphne Marlatt, Jack Hodgins, écrivains surtout des Prairies et de la Côte ouest qui ont transformé le paysage littéraire canadien de ces dix dernières années; ni de passer sous silence Sheila Watson, Alice Munro, Audrey Thomas dont les fictions sont toujours des explorations du langage. Mais il reste que dans le contexte canadien-anglais les expériences sur la langue, les tentatives de déconstruction sont greffées sur un fond de réalisme qui se différencie nettement de la tradition littéraire québécoise. Selon Gilles Marcotte, l'absence presque totale de réalisme dans le roman québécois correspond à l'absence de l'Histoire, caractéristique des peuples colonisés : «Le roman est privé d'intrigue, comme la société est privée d'action, d'histoire; il ne se passe rien dans le roman, parce qu'il ne se passe rien dans la société » 17. Le paradoxe de la situation du Canadien anglais est qu'il est à la fois colonisateur et colonisé, et que malgré des crises cilturelles cycliques concernant son existence par rapport à la culture américaine, la littérature canadienne-anglaise est traversée par une conscience de l'Histoire comme processus et possibilité.

Cette tradition que j'appelle «réaliste» et dont l'œuvre de Margaret Laurence est l'exemple moderne le plus frappant inclut d'autres écrivains majeurs comme Robertson Davies, Timothy Findley et Margaret Atwood, ainsi que des auteures franchement féministes comme Aritha Van Herk et Joan Barfoot. Mise en contraste avec une tradition québécoise caractérisée par «autre chose» - comment le nommer? l'utopie? le désespoir? la révolution? le désir? la fascination du langage? - la tradition réaliste me semble un domaine privilégié d'analyse si nous voulons explorer de façon valable les rapports entre les deux traditions culturelles.

17. Le Roman à l'imparfait: Essais sur le roman québécois d'aujourd'hui. Montréal, Editions La Presse, Coll, "Échanges», 1976, p. 27. 
Par réalisme, je ne veux pas simplement dire une attitude face à la structure narrative ou au langage qui, malgré les interrogations et l'expérimentation, demeurent fondamentalement intacts dans la fiction canadienne-anglaise, mais aussi ce rapport optimiste et souvent lié à une idéologie de gauche face à la société, qui est présent dans presque toute l'écriture canadienne-anglaise. Comme l'idéologie «Red Tory» (conservateur de gauche) qui a été si importante dans le développement d'une culture canadienne distincte de l'américaine (basée en grande partie sur l'entreprise publique), le réalisme littéraire canadien-anglais est un fascinant mélange d'éléments conservateurs et radicaux.

Le contraste avec la tradition québécoise est tout à fait évident quant on compare, par exemple, les romans de Margaret Atwood avec ceux d'Hubert Aquin ou de Marie-Claire Blais. Chez Atwood - et ceci malgré son exposé lucide et ironique de l'injustice sexuelle et sociale est sa conscience des jeux du langage - la vision, réaliste et essentiellement moralisatrice, est celle d'une société perfectible; tandis que chez Aquin et Blais, la vision, plus extrême et passionnée, aboutit nécessairement à la révolution totale ou à la mort.

Les mêmes contrastes structuraux et idéologiques seraient possibles entre la plupart des grands écrivains des deux traditions. Dans l'œuvre de poètes tels Charles G.D. Roberts, Archibald Lampman, F.R. Scott, Dorothy Livesay, Earle Birney et Al Purdy, il y a un engagement social explicite et la langue, qui ne fait pas question, est tout au plus utilisée comme moyen de décrire la réalité; par contre, chez Émile Nelligan, SaintDenys Garneau ou même chez un poète engagé comme Gaston Miron, on dirait que la préoccupation première n'est pas la réalité mais le voyage dans le langage. À cet égard il faudrait mentionner la présence dans la poésie canadienne-anglaise d'un humour qui est à la fois le signe d'une acceptation confiante du réel et un outil pour sa transformation. Déjà dans les années 1950 un poète féministe comme Livesay peut incarner les principes masculin et féminin dans l'image ironique de «Bartok and the Geranium », montrant le triomphe de l'endurance et de la jouissance féminines du géranium sur la passion frénétique de la mâle musique de Bartok:

$\mathrm{He}$ in a mad intensity

Soars beyond sight

Then hurls, lost Lucifer,

From heaven's height,

And when he's done, he's out:

She lays a lip against the glass

And preens herself in light 18.

18. Dorothy Livesay - Selected Poems (1926-1956), Toronto, Ryerson Press, 1957, p. 73. 
Est-ce uniquement un hasard si le paysage est une catégorie essentielle de la poésie et de la critique littéraire canadiennes-anglaises 19 alors que ce n'est pas le cas dans la tradition littéraire du Québec? Qu'elle ait inspiré la terreur ou l'amour, ou que l'on y voie une force qui peut instruire, la nature a existé dans la poésie canadienne-anglaise comme une réalité extérieure au poète, alors qu'au Québec elle a été davantage la projection symbolique de soi ou de la quête pour une identité nationale. Une observation de Jacques Godbout aidera à préciser ce rapport entre paysage et réalisme: selon Godbout, si l'on demandait au Canadien anglais "typique» de choisir un tableau qui représentait sa culture, il en choisirait un du Groupe des Sept (c'est-à-dire une représentation du paysage), tandis que le Québécois choisirait un tableau de Jean-Paul Lemieux - la représentation en gros plan d'un individu solitaire et en arrière-plan un paysage nu et désolé.

Malgré le parallélisme établi entre nos deux univers romanesques par des critiques tels Ronald Sutherland - du milieu rural à la grande ville, de l'aliénation à la libération - je crois que les différences fondées sur les attitudes face à la réalité - et ici je veux dire la réalité sociale - sont tout aussi évidentes entre nos deux traditions. À la différence du roman de la terre québécois, fermé et circulaire dans sa structure et implacablement patriarcal dans son système de valeurs, le roman de la terre au Canada anglais paraît avoir une structure ouverte et une vision qui s'accommode des changements sociaux et technologiques. Abe Spalding dans Fruits of the Earth de Frederick Philip Grove est évidemment un patriarche, mais au cours du roman il sera assagi et transformé par la rébellion de sa fille et par la modernisation nécessaire de son mode de vie agricole; le cultivateur québécois, par contre, s'accroche à sa tradition patriarcale, catholique et canadiennefrançaise et en voyant son monde menacé, est acculé à la défaite plutôt qu'au compromis. Même un roman comme As For Me and My House de Sinclair Ross, souvent considéré comme typique de la victimisation du protagoniste canadien-anglais face a la nature et une société hostile, semble ouvert dans sa structure narrative et dans sa vision du couple et de la société lorsqu'il est comparé à un roman de la même époque et de la même configuration sociologique, Poussière sur la ville d'André Langevin. Les ambiguïtés du roman de Ross ont été beaucoup débattues par les critiques, mais il reste que la narration y est confiée à une femme, l'épouse du protagoniste, et que malgré l'incommunication à l'intérieur du couple qui fait le sujet de la narration, elle conclut par l'espoir d'un dialogue renouvelé entre femme et mari et la possibilité de quitter la petite ville fermée où ont eu lieu leurs malheurs pour aller recommencer ailleurs. Dans le roman de Langevin, par contre, l'épouse du narrateur, vue uniquement de l'extérieur et de son point de vue à lui, pose par son suicide le geste absolu du nécessaire

19. Le débat sur la configuration du paysage et sur les "archétypes» qui s'en dégagent est central dans des ouvrages critiques aussi marquants que la conclusion au Literary History of Canada de Northrop Frye, Toronto, University of Toronto Press, 1965, Survival de Margaret Atwood, Toronto, Anansi, 1971 et Butterfly on Rock de D.G. Jones, Toronto, Unviersity of Toronto Press, 1970. 
éclatement d'un ordre fermé, et le narrateur reste dans la petite ville hypocrite, conscient que sa lutte ne peut pas se poursuivre ailleurs que dans cet espace fermé. De tels contrastes, basés sur la façon dont l'espace idéologique de chaque culture a influencé ses structures littéraires, aideraient à expliquer pourquoi les femmes semblent avoir eu davantage d'espace pour exister en tant qu'individus autonomes dans la tradition canadienneanglaise que dans la québécoise, où dès le dix-neuvième siècle les femmesécrivains semblent tenter, à travers les mots, de faire éclater les murs d'une prison.

En examinant les différentes traditions philosophiques qui ont modelé les cultures du Canada anglais et du Québec, nous découvririons une dimension supplémentaire et une explication de ces contrastes fondamentaux. Réduits à leur niveau le plus simple, ces contrastes seraient résumés dans la devise célèbre de Pierre Elliott-Trudeau, «La raison avant la passion » (ou le Canada avant le Québec). De fait, il y a eu dans la tradition culturelle canadienne-anglaise une foi démesurée dans la raison, dans les possibilités de la négociation, dans la nature à la fois progressive et conservatrice d'une tradition qui croit que la réalité peut être gérée, changée et ouverte sur l'avenir 20. Du côté québécois, certainement à cause du rapport colonisateurcolonisé et aussi à cause du caractère absolutiste de la tradition catholique, il semble y avoir eu au contraire un refus ou une incapacité de transiger avec le réel. L'espace est fondamentalement habitable dans la littérature canadienne-anglaise tandis que dans la littérature du Québec c'est une prison qu'il faut faire éclater par les jeux du langage et de l'imaginaire. Estce pure coïncidence qu'en plus des différences d'idéologies, de traditions religieuses et de circonstances historiques, il y a une différence fondamentale dans l'espace réel habité par ces deux littératures? L'espace géographique de la littérature traditionnelle du Québec, enfermé et diminué, est perçu comme une version avilie de l'immense canevas géographique occupé par la Nouvelle France avant la Conquête. L'espace dans la littérature canadienne-anglaise est ouvert, éclectique et souvent déroutant dans sa disparité régionale: c'est un espace qui permet l'exploration et l'expansion. Contrairement au Québécois, le Canadien anglais a eu le loisir d'être «de passage», comme le dit le poète $\mathrm{Al}$ Purdy, «courant et courant sans jamais quitter son chez-soi»:

\author{
Riding the boxcars out of Winnipeg in a morning \\ after rain so close to \\ the violent sway of fields it's \\ like running and running \\ naked with summer in your mouth...
}

20. Voir Leslie Armour et Elizabeth Trott - The Faces of Reason: An Essay on Philosophy and Culture in English Canada 1850-1950, Waterloo, Wilfrid Laurier University Press, 1981. 


\begin{abstract}
After a while there is no arrival and no departure possible any more you are where you were always going and the shape of home is under your fingernails 21 .
\end{abstract}

Pour revenir à l'image d'Hubert Aquin dans Prochain épisode, le Canadien anglais prend pour acquis «le plaisir d'habiter une maison", alors que le Québécois, toujours à la poursuite de son «exil chaotique dans des hôtels (qu'il) n'habite jamais ", a appris à défier les structures du réel et à saisir la langue pour la faire sienne.

En conclusion, je suis tentée par le parallélisme rassurant et la symétrie des mouvements que je crois présents dans un grand nombre des écritures les plus récentes du Canada anglais et du Québec: dans la littérature canadienne-anglaise, surtout celle de la Côte ouest et des Prairies et celle de certaines féministes, la tendance à s'écarter d'un certain réalisme pour habiter de façon plus confiante les domaines de la fiction, de l'imaginaire et du langage; en même temps, dans la littérature québécoise, le fait que dans la poésie et les fictions d'écrivains tels que France Théoret, Philippe Haeck et Nicole Brossard, le réel est perceptible comme jamais auparavant. J'aimerais cependant ajouter une dernière réflexion sur la question du réalisme, qui est à mon avis une question centrale et non résolue pour les écrivains et les critiques du Canada anglais. Le mot «réalisme» est mal accueilli dans les cercles littéraires modernes, et il est certain qu'il peut effectivement représenter un espace littéraire et idéologique trop confortable et conventionnel. Aritha Van Herk soutient dans un article récent, et de façon convaincante, que les écrivain(e)s du Canada doivent «dé-sirer»* le réalisme et se libérer de l'emprise de l'histoire pour laisser davantage la place au désir et à l'audace de créer des fictions non mimétiques 22. Dans les échanges entre écrivaines et critiques féministes des deux cultures, les Canadiennes-anglaises tendent de plus en plus vers la conclusion que la société patriarcale et la tradition du réalisme littéraire ont été une prison pour les femmes en quête d'un langage et d'un avenir nouveaux, et que les écrivaines du Québec ont beaucoup à leur apprendre sur les femmes et le langage.

* Jeu de mots intraduisible sur «desire» et «de-sire» (dés-engendrer; du verbe sire, engendrer). NDLR

21. Al Purdy, "Transient", dans Selected Poems, Toronto, McClelland and Stewart 1972, p. 122-3.

22. "Desire in Fiction: De-Siring Realism", Dandelion Vol. VIII No 2; Fall-Winter 1981-2, p. 32-8. 
Mais si le réalisme et ce que Dorothy Livesay appelle la «tradition documentaire 23 " ont joui d'une place centrale dans, le patrimoine culturel canadien-anglais, si le réalisme idéologique nous a fourni, même en tant que femmes, un espace pour exister et évoluer, le réalisme et l'histoire continueront, et devront continuer, à être intégrés à nos désirs et à nos fictions. Quelles que soient les mutations effectuées par les expériences langagières des modernes et des féministes, les écrivain(e)s du Québec et du Canada anglais continueront à habiter leur territoire respectif en fonction de leur propre imaginaire lequel, il faut le souhaiter, sera peut-être spéculaire mais sûrement pas le calque de l'autre.

23. "The Documentary Poem : a Canadian Genre", in Contexts of Canadian Criticism, ed. Eli Mandel, Toronto, University of Toronto Press, 1971, p. 267-81. 